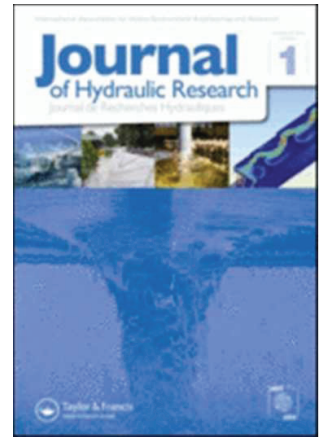

\title{
Comparison of wetting and drying numerical treatments for the Roe Riemann scheme
}

\begin{tabular}{|c|c|}
\hline Journal: & Journal of Hydraulic Research \\
\hline Manuscript ID & TJHR-2015-0215.R3 \\
\hline Manuscript Type: & Research paper \\
\hline Date Submitted by the Author: & 18-Nov-2016 \\
\hline Complete List of Authors: & $\begin{array}{l}\text { Martins, Ricardo; The University of Sheffield, Department of Civil and } \\
\text { Structural Engineering; MARE - Marine and Environmental Sciences Centre, } \\
\text { Department of Civil Engineering, FCT, University of Coimbra; IMAR - } \\
\text { Institute of Marine Research, Department of Civil Engineering, FCT, } \\
\text { University of Coimbra } \\
\text { Leandro, Jorge; Universidade de Coimbra Faculdade de Ciencias e } \\
\text { Tecnologia, Departamento de Engenharia Civil; MARE - Marine and } \\
\text { Environmental Sciences Centre, Department of Civil Engineering; IMAR - } \\
\text { Institute of Marine Research, Department of Civil Engineering } \\
\text { Djordjevic, Slobodan; University of Exeter }\end{array}$ \\
\hline Keywords: & $\begin{array}{l}\text { Flood Modelling, Local Inertial Equations, Overland Flows, Shallow Flows, } \\
\text { Shallow Water Equations, Wetting-Drying Fronts, Roe Riemann Scheme }\end{array}$ \\
\hline JHR Keywords: & $\begin{array}{l}\text { Two- dimensional models < Computational methods in hydro-environment } \\
\text { research and fluid dynamics, Flood modeling }<\text { Applied fluid mechanics and } \\
\text { hydraulic engineering, Overland flows }<\text { Environmental Fluid Mechanics, } \\
\text { Shallow flows }<\text { Environmental Fluid Mechanics }\end{array}$ \\
\hline \multicolumn{2}{|c|}{$\begin{array}{l}\text { Note: The following files were submitted by the author for peer review, but cannot be converted to } \\
\text { PDF. You must view these files (e.g. movies) online. }\end{array}$} \\
\hline $\begin{array}{l}\text { JHR.tex } \\
\text { Biblio.bib }\end{array}$ & \\
\hline
\end{tabular}




\section{SCHOLARONE $^{\text {Tw }}$ \\ Manuscripts}

2

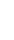
9
10 
To appear in the Journal of Hydraulic Research

Vol. 00, No. 00, Month 20XX, 1-18

Research Paper

\title{
Comparison of wetting and drying numerical treatments for the Roe Riemann scheme
}

\author{
RICARDO MARTINS, Research Associate, Department of Civil and Structural Engineering, The University of \\ Sheffield., Mappin Street, Sheffield S1 3JD, South Yorkshire, United Kingdom \& MARE - Marine and Environmental \\ Sciences Centre, Department of Civil Engineering, FCT, University of Coimbra, 3004-517 Coimbra, Portugal \\ Email: Ricardo.Martins@sheffield.ac.uk(author for correspondence)
}

JORGE LEANDRO, Lecturer, Department of Civil, Geo and Environmental Engineering, Chair of Hydrology and River Basin Management, Technical University of Munich, Arcisstrasse 21, 80333 Munich, Germany \& MARE Marine and Environmental Sciences Centre, Department of Civil Engineering, FCT, University of Coimbra, 3004-517 Coimbra, Portugal

Email: jorge.leandro@tum.de

SLOBODAN DJORDJEVIĆ (IAHR Member), Professor, Centre for Water Systems, University of Exeter, North Park Road, Exeter EX4 4QF, United Kingdom

Email:S.Djordjevic@exeter.ac.uk

\begin{abstract}
Accurate characterization of wetting-drying fronts in free surface flows is challenging because it is difficult and computationally demanding to track the exact position of the interface. This work presents a novel numerical treatment of the wetting-drying fronts applied to an approximate Roe Riemann solver and compares it to four other approaches. The numerical treatments were implemented both for the shallow water equations and for the local inertial equations. The results of this comparison overall showed a good agreement. For the tests conducted it was verified that element removal numerical treatments with global distributing of water can introduce errors and degenerate the solution introducing or displacing water upstream. Local correction and flux restricting numerical treatments show the best results. The negative depth numerical treatments provided similar results to the local correction and flux restricting numerical treatment, although with mass conservation errors.
\end{abstract}

Keywords: Flood Modelling, Local Inertial Equations, Overland Flows, Shallow Flows, Shallow Water Equations, Wetting-Drying Fronts

\section{Introduction}

The characterisation of wetting-drying (WD) fronts in free surface flows is often demanding because the exact position of the interface is difficult to calculate or the process to obtain it is computationally demanding. In technical literature WD fronts have been treated using several different numerical approaches.

Medeiros and Hagen (2013) present a review of numerical treatments and divide them into four types. Two more approaches can be added to this classification, resulting in six categories: (1) Thin Film, (2) Element removal, (3) Depth extrapolation, (4) Artificial porosity (also termed negative depth in Medeiros and Hagen (2013)), (5) Positivity-Preserving, and (6) Flux Correctors.

Thin layer numerical treatments rely on the addition and subtraction of a thin layer of water to the computational nodes, preserving positivity permanently. These treatments produce, however, a spurious water surface slope and an artificial pressure gradient (Heniche, Secretan, Boudreau, \& Leclerc, 2000) and may introduce oscillations and numerical instabilities (Kärnä et al., 2011). This approach also implies that the entire domain is always calculated, thus increasing the computational time (Medeiros \& Hagen, 2013). 
Element removal numerical treatments are very common (Medeiros \& Hagen, 2013). They rely on the algorithm that checks if a node is included in the computational domain or not (Nikolos \& Delis, 2009). The simple implementation process makes it very attractive and computationally effective. Usually a threshold is defined that turns the computational cell "on" or "off". After such verification, the numerical treatment redistributes the volume. This process is fairly straightforward for first order methods. The major disadvantages of such approach are its unsuitability for implicit or semi-implicit methods, large sensitivity to round-off errors (Hof \& Vollebregt, 2005), possibility of rapid toggling of computational cells, and dampening of overland flow (Medeiros \& Hagen, 2013).

Depth extrapolation and Wave-front tracking numerical treatments (Aureli, Maranzoni, Mignosa, \& Ziveri, 2008) are very accurate but difficult to implement. They rely either on extrapolation of the free surface in the computational cell or on pinpointing the exact location of the wetting-drying front. Techniques such as VFR (Volume/free-surface relation) have improved the accuracy of the wave front tracking (Begnudelli \& Sanders, 2006).

Artificial porosity approach consists of adding a porous layer, circumventing some of the disadvantages of the previous numerical treatments (Hof \& Vollebregt, 2005). This numerical treatment however requires that artificial porosity is applied not only to negative depths but also to a small depth above the bed elevation.

Another class of numerical treatments are positivity-preserving numerical treatments - Xing and Shu (2011) that relies on a positivity preserver limiter, and Duran, Liang, and Marche (2013) that uses nonconservative variables, are examples of such numerical treatments. Generally they conserve mass, globally and locally, converge to the analytical solution on grid independent problems and have an analytical CFL condition that ensures the numerical stability. Other examples of these numerical treatments are the exact Riemann solver (Godunov, Zabrodin, \& Prokopov, 1961), HLLE (Einfeldt, Munz, Roe, \& Sjögreen, 1991), Kinetic (Audusse \& Bristeau, 2005), VFRoe (Buffard, Gallouët, \& Hérard, 2000), and the numerical treatment proposed by Murillo and García-Navarro (2010).

The ultimate family of numerical treatments is termed flux corrector or flux restricting. Leandro, Chen, and Schumann (2014) developed a treatment for flood inundation that limits the inter-cell flux such that a cell is never depleted. The authors applied it to the diffusive wave model and showed it to be stable also for urbanized areas (Leandro, Schumann, \& Pfister, 2016). This numerical treatment is easily used for a first order diffusive wave model, however with the introduction of second or higher order reconstructions in FVM it may become more difficult to apply. First order methods can benefit from this numerical treatment. Other examples are presented by Brufau, García-Navarro, and Vázquez-Cendón (2004) who applied flux corrector to a Roe Riemann scheme where the wave-strengths and fluxes are redefined "a priori" so that the mass conservation is guaranteed, and Murillo, García-Navarro, Burguete, and Brufau (2006) who redefined the bottom slopes in a way that is equivalent to redistributing the updating fluxes.

Without correction the wetting-drying front numerical treatments usually produce spurious oscillations or violate mass conservation when depth becomes negative. Some authors deem a small mass continuity error acceptable (Bates \& Hervouet, 1999; Neal et al., 2012). The aim of this work is to provide a comparison between five numerical treatments that apply corrections after the time step calculation is performed. In this paper we propose a novel element removal wetting-drying numerical treatment (section 2.2), based upon the concept of gravitational attraction. In addition to being globally mass conservative, this numerical treatment is also "locally" conservative limiting the exchanges to an adjacent cell. In order to verify the accuracy of this numerical treatment, a thorough comparison between the novel numerical treatment and four (sections 2.2) well established ones existent in technical literature is made. Finally the differences between all models are analysed and discussed (section 3) and conclusions drawn regarding the numerical treatments used (section 4) in this paper for both the Shallow Water Equations and the Local Inertial Equations.

\section{Numerical models of overland flow}

The wetting-drying numerical treatments were tested on a Finite Volume Roe Riemann solver applied to two sets of equations, namely the shallow water equations and the local inertial equations. 


\subsection{Hydrodynamic models}

The generic conservation law is given by the mass conservation equation and the momentum conservation equations in $x$ and $y$ direction:

$$
\frac{\partial}{\partial t} \boldsymbol{U}+\nabla \cdot \mathbf{W}(\boldsymbol{U}, x, y)-\boldsymbol{S}(\boldsymbol{U}, x, y)=0
$$

When using the Manning friction term to model the bed friction stress, the matrix form of the third term on the left hand side becomes common to the two models used here and is therefore:

$$
\boldsymbol{S}(\boldsymbol{U}, x, y)=\left(\begin{array}{c}
0 \\
-\left(g h B_{x}-u g n^{2}\|\boldsymbol{w}\| h^{-1 / 3}\right) \\
-\left(g h B_{y}-v g n^{2}\|\boldsymbol{w}\| h^{-1 / 3}\right)
\end{array}\right)
$$

where $h$ is water depth, $\boldsymbol{w}$ is velocity vector, composed of $u$ and $v$ that are the velocity components in the $x$ and $y$ direction respectively, $g$ is gravitational acceleration, $B_{x}$ and $B_{y}$ are the bed slope in the $x$ and $y$ direction respectively.

\section{Shallow Water Equations}

In matrix form, for the Shallow Water equations, the expanded terms become:

$$
\begin{gathered}
\boldsymbol{U}=\left(\begin{array}{c}
h \\
u h \\
v h
\end{array}\right) \\
\mathbf{W}(\boldsymbol{U}, x, y)=\left(\begin{array}{cc}
u h & v h \\
\frac{1}{2} g h^{2} & u^{2} h+u v h \\
v^{2} h+u v h & \frac{1}{2} g h^{2}
\end{array}\right)
\end{gathered}
$$

The domain is divided using a node-centred unstructured triangular mesh (Nikolos \& Delis, 2009), with staggered variables. The numerical fluxes are calculated using an upwind first order in time and space well balanced Roe Riemann solver (Nikolos \& Delis, 2009). The bed elevation flux is well balanced using the Extended C-property thus achieving a perfect balance in hydrostatic conditions (Castro et al., 2005) and the velocities used in the wetting-drying front are computed as proposed by Brufau, Vázquez-Cendón, and García-Navarro (2002). Entropy is enforced by the use of the entropy fix scheme by Harten (1983). The friction source term is computed using a semi-implicit pointwise Runge-Kutta method (Liang \& Marche, 2009). The Roe scheme is not a positivity-preserving scheme and therefore an "a priori" analytically deducted CFL condition cannot be defined, however, and in order to keep the scheme stable the CFL condition used in the numerical calculations is the one presented in Nikolos and Delis (2009) for the SWE and Martins, Leandro, and Djordjević (2015) for the GWM.

\section{Local Inertial Equations}

The second model is the Gravity Wave Model (GWM) is based upon the non-linear local inertial equations. This set of equations neglects the convective acceleration terms when compared to the SWE. In the matrix 
conservative form:

$$
\begin{aligned}
\boldsymbol{U} & =\left(\begin{array}{c}
h \\
u h \\
v h
\end{array}\right) \\
\mathbf{W}(\boldsymbol{U}, x, y) & =\left(\begin{array}{cc}
u h & v h \\
\frac{1}{2} g h^{2} & 0 \\
0 & \frac{1}{2} g h^{2}
\end{array}\right)
\end{aligned}
$$

A similar procedure to the one used for SWE is used to integrate Equations (5) and (6). It involves a first order in space and time finite volume method (Martins et al., 2015) with a spatial 2D node-centred staggered unstructured triangular mesh. Integration in space is divided in two steps - the numerical fluxes and the bed elevation fluxes. The former are transformed from an area integral to a curve integral through the use of Gauss divergence theorem that accounts for the fluxes over the boundaries of the cell. By creating a Riemann problem between two generic adjacent points the inter-cell fluxes are then evaluated using a Roe approximate Riemann solver with averaged values of the primitive variables. The bed elevation fluxes are calculated using an upwind method derived specifically for this set of equations and this numerical method by respecting the extended C-property (Castro et al., 2005) with the velocity for the wetting-drying fronts as given by Brufau et al. (2002), thus avoiding spurious oscillations. This is achieved by projecting the source term onto the eigenvectors (i.e. the wave-strengths) of the Jacobian of the fluxes. Once linearised it is evaluated at the same state as the inter-cell fluxes. Bed friction is calculated using Manning's equations and is computed with a point-wise semi implicit method (Song, Zhou, Guo, Zou, \& Liu, 2011). The time integral is an explicit first order Euler method with an increment of time controlled by the CFL condition. More details about this model can be found in Martins et al. (2015).

\section{Discrete notation of the numerical schemes}

Both systems (3), (4) and (5), (6) are solved using the Roe solver presented in detail by Martins et al. (2015) using a node centred triangular cell. The conservative discrete form on a mesh computational cell is:

$$
\boldsymbol{U}_{P}^{t+1}=\boldsymbol{U}_{P}^{t}+\frac{\Delta t}{A_{P}}\left(\sum_{\forall Q \in K n_{P}} \psi_{P Q}^{t}-\sum_{\forall Q \in \boldsymbol{K} n_{P}} \phi_{P Q}^{t}-\sum_{\forall Q \in \boldsymbol{K} \boldsymbol{n}_{P}} \phi_{P Q}^{t} \text { Out }\right)+\mathbf{S}_{\mathbf{f}}\left(\mathbf{U}_{\mathbf{P}}\right)^{t}
$$

where $P$ is a generic cell represented by its centre, $Q$ is a generic adjacent neighbour across the set of neighbour points $\boldsymbol{K} \boldsymbol{n}_{P}$ of $P, A_{P}$ the area of cell $P, \phi_{P Q}^{t}, \phi_{P Q}^{t}$ Out and $\psi_{P Q}^{t}$ are the numerical fluxes evaluated based on the upwind Roe solver at time step ${ }^{t}$.

\subsection{Wetting-drying numerical treatments}

Five numerical treatments are tested. In SWE and GWM simulation a threshold value $\left(\varepsilon_{w d}\right)$ is often used instead of zero to limit negative depths and spurious velocities. For the sake of comparability the value used herein is $10^{-5}(\mathrm{~m})$. Table 1 presents a summary of the characteristics of the WD numerical treatments used.

\section{Element removal numerical treatment - ERI}

ER1 is an element removal numerical treatment that corrects depth to zero in a cell whenever it becomes lower than a threshold depth, thus avoiding negative depths and some spurious velocity values, and allowing 
Table 1 Summary of the tested WD numerical treatments' characteristics regarding mass conservation and if only negative depths are corrected or is $\varepsilon_{w d}$ higher than zero

\begin{tabular}{|c|c|c|c|}
\hline numerical treatment & $\begin{array}{l}\text { Global Mass } \\
\text { Conservation }\end{array}$ & $\begin{array}{l}\text { Local Mass } \\
\text { Conservation }\end{array}$ & $\begin{array}{c}\text { Only negative } \\
\text { depths }\end{array}$ \\
\hline ER1 & No & No & Yes \\
\hline ER2 & Yes & No & No \\
\hline ER3 & Yes & Yes $^{\mathrm{a}}$ & No \\
\hline P1 & No & No & Yes \\
\hline FR1 & Yes & Yes/No ${ }^{b}$ & Yes/No ${ }^{b}$ \\
\hline
\end{tabular}

computation of the next time step (i.e. $h_{P}>0 \forall P$ ). The equations used are:

$$
\begin{gathered}
h_{P}=\left\{\begin{array}{ccc}
h_{P} & \text { if } & h_{P} \geq \varepsilon_{w d} \\
0 & \text { if } & h_{P}<\varepsilon_{w d}
\end{array}\right. \\
\boldsymbol{w}_{P}=\left\{\begin{array}{cll}
\boldsymbol{w}_{P} & \text { if } & h_{P} \geq \varepsilon_{w d} \\
0 & \text { if } & h_{P}<\varepsilon_{w d}
\end{array}\right.
\end{gathered}
$$

This treatment eliminates negative depth cells, however it does not preserve mass nor momentum and can therefore introduce large errors in the model if not kept within reasonable limits. A way to do so is through reduction of CFL number, and therefore $\Delta t$. This process, however, might result in an exponential increase of computational time.

\section{Element removal numerical treatment - ER2}

ER2 is an element removal numerical treatment based on ER1, however instead of just correcting the depth it insures a global mass continuity. Computationally, ER1 is applied to identify wet and dry cells, followed by an algorithm that redistributes the volume removed or added to each cell by the full domain according to the volume in each cell. Nikolos and Delis (2009) apply a similar numerical treatment however the volume is redistributed uniformly all over the domain. First, for each point:

$$
\left\{\begin{array}{lll}
V_{P_{W e t}}=A_{P} h_{P} & \text { if } & \varepsilon_{w d} \leq h_{P} \\
V_{P_{D r y^{-}}}=A_{P}\left|h_{P}\right| & \text { if } & h_{P}<0 \\
V_{P_{\text {Dry }}+}=A_{P} h_{P} & \text { if } & 0<h_{P}<\varepsilon_{w d}
\end{array}\right.
$$

\section{Followed by:}

$$
h_{P}=\left\{\begin{array}{cll}
h_{P} & \text { if } & h_{P} \geq \varepsilon_{w d} \\
0 & \text { if } & h_{P}<\varepsilon_{w d}
\end{array}\right.
$$

Correcting the depth to:

$$
h_{P}^{w d}=h_{P}\left(1+\frac{\sum_{P=1}^{N}\left(V_{P_{D r y}+}-V_{P_{D r y^{-}}}\right)}{\sum_{P=1}^{N} V_{P_{W e t}}}\right)
$$


where $h_{P}$ is the depth at point $P, V_{P_{W e t}}$ is the total volume of water above the threshold at point $P, V_{P_{D r y}+}$ is the total volume of water below the threshold but above 0 , and $V_{P_{D r y^{-}}}$is the volume for negative water depths. The momentum suffers no correction.

\section{Element removal numerical treatment - ER3}

ER3 is a novel numerical treatment proposed herein. It is based on the concept of gravitational attraction and relies on two variables (proximity and volume) to redistribute water along neighbour cells. The neighbourhood degree of proximity can be defined as an input parameter along with "gravitational" constants.

The algorithm defines a cell as wet or dry by setting variable $W e t_{P}$ as 0 or 1 if the depth is lower or higher than the wetting-drying threshold $\left(\varepsilon_{w d}\right)$, respectively. The volume in each cell $V_{P}$ is calculated and the original depths stored in new variables, higher $\left(h_{P}^{w}\right)$ and lower $\left(h_{P}^{w d}\right)$ than the threshold $\left(\varepsilon_{w d}\right)$ :

$$
\begin{gathered}
\text { Wet }_{P}=\left\{\begin{array}{lll}
0 & \text { if } & h_{P}<\varepsilon_{w d} \\
1 & \text { if } & h_{P} \geq \varepsilon_{w d}
\end{array}\right. \\
V_{P}=h_{P} A_{P} \\
h_{P}^{w}=\left\{\begin{array}{lll}
0 & \text { if } & h_{P}<\varepsilon_{w d} \\
h_{P} & \text { if } & h_{P} \geq \varepsilon_{w d}
\end{array}\right. \\
h_{P}^{w d}=\left\{\begin{array}{lll}
h_{P} & \text { if } & h_{P}<\varepsilon_{w d} \\
0 & \text { if } & h_{P} \geq \varepsilon_{w d}
\end{array}\right.
\end{gathered}
$$

The maximum $(M V)$ and minimum $(m V)$ volumes for the time step in the domain are computed along with the smallest $(m D)$ and largest $(M D)$ distance between cell centres:

$$
m V=\min \left(V_{P}\right), \quad M V=\max \left(V_{P}\right), \quad m D=\min \left(D_{P Q}\right), \quad M D=\max \left(D_{P Q}\right)
$$

A "gravitational" attraction coefficient for the interaction between each pair of adjacent cells $\left(G_{P, Q}\right)$ is calculated using the proximity and volume of each neighbour cell:

$$
G_{P, Q}=C_{V_{w d}}\left(1-\frac{V_{Q}-m V}{m V-M V}\right)^{\alpha_{V_{w d}}}+C_{D_{w d}}\left(\frac{D_{P Q}-M D}{m D-M D}+1\right)^{\alpha_{D_{w d}}}
$$

Volume "gravitational" attraction increases with volume in the neighbourhood cell while distance "gravitational" attraction is reduced with distance. Equation (17) implies that as the distance diminishes and the volume increases the volume received becomes higher. Adjustments of the exchanged volume are allowed with the introduction of four coefficients defined at the beginning of simulation: $C_{V_{w d}}, C_{D_{w d}}, \alpha_{V_{w d}}, \alpha_{D_{w d}}$. $C_{V_{w d}}$ and $C_{D_{w d}}$ control the linear proportionality weighting of the distribution relating to the volume "gravitational" attraction $\left(V_{w d}\right)$ or distance "gravitational" attraction $\left(D_{w d}\right) . \alpha_{V_{w d}}$ and $\alpha_{D_{w d}}$ control the amount of linearity, allowing for a non-linear distribution.

This "gravitational" coefficient can be adjusted to one degree of proximity or two (i.e. only the neighbourhood cells accept volume, or the neighbourhood of the neighbourhood cell accept volume transfer). This numerical treatment guarantees that the negative or below threshold cell only contributes volume to the adjacent neighbour in case one degree is selected. 
The coefficients are normalized during the computations, therefore, there are no range restrictions. Each cell has a total amount of neighbour "gravitational" attraction that is calculated using:

$$
T G_{P}=\sum_{Q=1}^{N_{Q}} G_{P, Q}
$$

Each wet cell updates their neighbour cells as:

$$
h_{Q}^{w^{\prime}}=h_{Q}^{w}+V_{P} \frac{G_{P, Q}}{T G_{P} A_{Q}}
$$

In addition to being globally mass conservative, this numerical treatment is also "locally" conservative (up to the degree of proximity predefined) as water is kept within the neighbourhood of the cell.

\section{Porosity numerical treatment - P1}

$\mathrm{P} 1$ is a porosity numerical treatment. The algorithm assumes that a cell stays negative and is removed from the calculations until the depth is again greater than zero with the negative depth stored in an auxiliary variable $\left(h_{P}^{n}\right)$. At the end of flux calculation, the cells have a trigger that defines if they are included in the next time step calculation or not $\left(W e t_{P}\right)$. If they have a depth lower than zero, for computational purposes it is temporarily adjusted to zero.

$$
\left\{\begin{array}{ccccc}
h_{P}=h_{P} & \text { and } & h_{P}^{n}=0 & \text { if } & h_{P} \geq 0 \\
h_{P}=0 & \text { and } & h_{P}^{n}=h_{P} & \text { if } & h_{P}<0
\end{array}\right.
$$

When the depth becomes larger than 0 the negative depth is removed from the new depth.

$$
\left\{\begin{array}{l}
h_{P}=h_{P}+h_{P}^{n} \quad \text { and } \quad h_{P}^{n}=0 \quad \text { if } \quad h_{P}+h_{P}^{n} \geq 0 \\
h_{P}^{n}=h_{P}+h_{P}^{n} \text { and } h_{P}=0 \quad \text { if } \quad h_{P}+h_{P}^{n}<0
\end{array}\right.
$$

\section{Flux restricting numerical treatment - FRI}

FR1 is based on the numerical treatment presented by Leandro et al. (2014), extended to GWM in Martins, Leandro, and Djordjević (2016) and herein for SWE. It implies a redefinition of the flux and can be considered a flux restricting, depth extrapolation numerical treatment or predictor-corrector. The computational steps are:

- Step 1: Calculation of the fluxes between all pair of cells: numerical fluxes $\left(\phi_{P Q}\right)$, source term fluxes $\left(\psi_{P Q}\right)$ and boundary fluxes $\left(\phi_{P Q}^{O u t}\right)$.

- Step 2: Sum of the fluxes in each cell: numerical fluxes $\left(\phi_{P}=\sum \phi_{P Q}\right)$, source term fluxes $\left(\psi_{P}=\right.$ $\left.\sum \psi_{P Q}\right)$ and boundary fluxes $\left(\phi_{P}^{B}=\sum \phi_{P Q}^{O u t}\right)$.

- Step 3: Calculating temporary depths from the fluxes by temporal integration $\left(\Phi_{P}=\left(\psi_{P}-\phi_{P}-\right.\right.$ $\left.\left.\phi_{P}^{O u t}\right) / A_{P}\right)$.

$$
h_{P}^{t+1}=h^{t}+\Delta t \Phi_{P}
$$

- Step 4: Tracing the cells to be recomputed based on the temporary depth $\left(h_{P}^{t+1}<0\right)$. 
- Step 5: Calculation of transfer coefficients between pairs of cells:

$$
\begin{gathered}
C_{P}=\frac{h_{P}}{\Delta t \Phi_{P}}, \quad C_{Q}=\frac{h_{Q}}{\Delta t \Phi_{Q}}, C_{P Q}=\frac{\min \left(h_{Q}, h_{P}\right)}{\Delta t\left(\psi_{P Q}-\phi_{P Q}-\phi_{P Q}^{O u t}\right)} \\
C_{P Q}^{f}=\min \left(C_{P}, C_{Q}, C_{P Q}, 1.0\right)
\end{gathered}
$$

- Step 6: Recomputing of fluxes in each cell:

$$
\phi_{P}=\sum C_{P Q}^{f} \phi_{P Q}, \quad \psi_{P}=\sum C_{P Q}^{f} \psi_{P Q}, \phi_{P}^{O u t}=\sum C_{P Q}^{f} \phi_{P Q}^{O u t}
$$

- Step 7: Calculating corrected depths and momentum.

- Step 8: Check if any cell is below $\varepsilon_{w d}$ to avoid spurious oscillations due to localized high velocities.

- Step 9: Apply ER2 numerical treatment if cell is below $\varepsilon_{w d}$.

\section{Numerical tests and discussion}

Four tests are performed: (1) wave propagation over an horizontal plane (comparison of the numerical treatments in slow moving waves) (2) non-breaking wave propagation over a planar beach (analysis of wetting-drying fronts/rears); (3) slow moving flood wave propagating over a 2D plain (radial symmetry); (4) valley flooding complex test following a rapid dam failure. All tests comprise an initial dry bed condition and the threshold constant is defined at $\varepsilon_{w d}=10^{-5}(\mathrm{~m})$. The CFL coefficient was set to 1 . The computational time (Intel Core i7 2630QM Processor) and average time step for each simulation $(\overline{\Delta t})$ are

\begin{tabular}{|c|c|c|c|c|c|c|c|c|c|c|c|}
\hline & Test & ER1 & ER2 & $\begin{array}{c}\text { GWM } \\
\text { ER3 }\end{array}$ & P1 & FR1 & ER1 & ER2 & $\begin{array}{l}\text { SWE } \\
\text { ER3 }\end{array}$ & P1 & FR1 \\
\hline \multirow{4}{*}{ 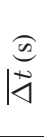 } & 1 & 1.486 & 1.486 & 1.486 & 1.486 & 1.486 & 1.213 & 1.213 & 1.213 & 1.213 & 1.213 \\
\hline & 2 & 1.203 & 1.202 & 1.194 & 1.201 & 1.189 & 0.967 & 0.966 & 0.966 & 0.967 & 0.966 \\
\hline & 3 & 2.223 & 2.223 & 2.223 & 2.223 & 2.223 & 1.612 & 1.612 & 1.612 & 1.612 & 1.612 \\
\hline & 4 & 3.018 & 4.174 & 4.215 & 4.204 & 4.092 & 2.807 & 3.790 & 3.855 & 3.845 & 4.092 \\
\hline (n) & 1 & 11 & 11 & 13 & 11 & 12 & 18 & 19 & 19 & 16 & 19 \\
\hline \pm & 2 & 36 & 36 & 50 & 40 & 49 & 133 & 134 & 167 & 157 & 164 \\
\hline$\vec{R}$ & 3 & 146 & 157 & 169 & 149 & 177 & 231 & 243 & 292 & 236 & 325 \\
\hline$\widetilde{U}$ & 4 & 119 & 76 & 116 & 81 & 136 & 131 & 160 & 175 & 224 & 176 \\
\hline
\end{tabular}
shown in Table 2.

Table 2 Average time steps and computational times for the simulations performed

\subsection{Non-breaking wave propagation over an horizontal plane}

The first test is the non-breaking wave propagation over an horizontal plane with friction. The test was first proposed by Hunter, Horritt, Bates, Wilson, and Werner (2005) and is performed in a 2D channel with constant width and constant friction thus rendering it a $1 \mathrm{D}$ problem with a flat bed. The test domain is 5000 $(\mathrm{m}) \times 400(\mathrm{~m})$ discretized as 5,273 points and 10,112 cells with an average edge of $21.48(\mathrm{~m})$ and was run with a Manning's friction constant of $0.03\left(\mathrm{~m}^{1 / 3} \mathrm{~s}^{-1}\right)$. The final simulation time was $3600(\mathrm{~s})$.

The test allows an analytical solution by assuming constant velocity in the test:

$$
\frac{\partial h}{\partial t}+u \frac{\partial h}{\partial x}=0
$$



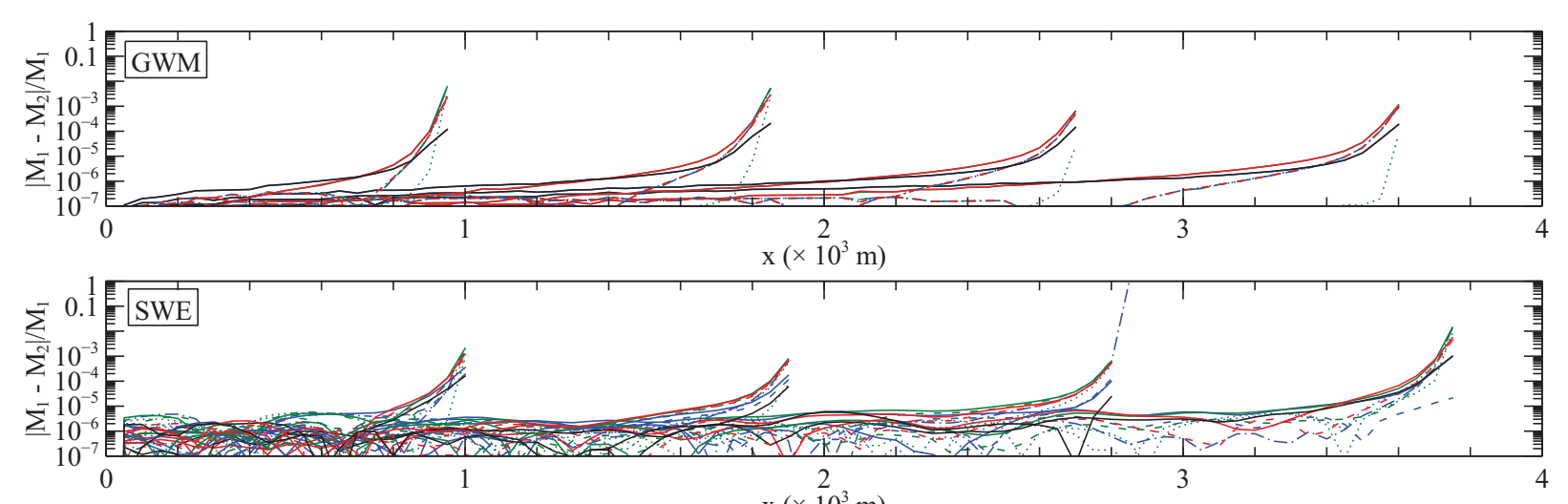

By integration of Equation (28) assuming that Equation (27) is the advection equation at constant velocity whose solution is $h(x, t)=h(x-u t, 0)$ the boundary condition for $x=0(\mathrm{~m})$ is:

$$
h(0, t)=\left(\frac{7}{3} n^{2} u^{3} t\right)^{3 / 7}
$$

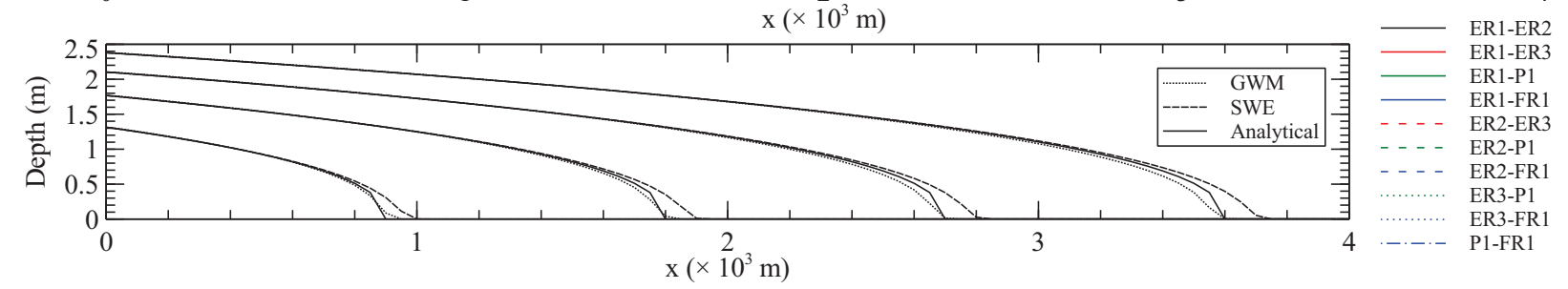

Figure 1 Non-breaking wave propagation over an horizontal plane for $t=900(\mathrm{~s}), t=1800$ (s), $t=2700$ (s) and $t=3600$ (s). Top: relative depth difference between numerical treatments using GWM; Centre: relative depth difference using SWE; Bottom: Free surface position for the analytical solution, GWM, and SWE.

Depth results for the $y$ centre profile are plotted in Fig. 1 - the top figure shows relative differences between each pair of WD numerical treatments for the GWM model, the centre figure displays the relative differences between SWE numerical treatments, and the bottom figure the simulated depth plotted for the average of all GWM and all SWE models. The average was chosen because the curves almost overlap and the differences between numerical treatments for the same model were not visible. Results are plotted for four times: $t=900$ (s), $t=1800$ (s), $t=2700$ (s) and $t=3600$ (s) sequentially.

Small differences in the results are seen since the threshold $\left(\varepsilon_{w d}\right)$ was not considered zero. This is particularly important in GWM WD were the absence of $\varepsilon_{w d}$ would result in an overlap of all results. This is because in a slow moving wave propagation, for the GWM the front wave speed is always the average between the celerities of the cells adjacent to the front wave, which means that the front wave is never faster than the perturbations travelling ahead of it. This is also valid to demonstrate that ER2 and FR1 share the same results for this test, as FR1 was not activated. Mass conservation is kept within machine precision for all numerical treatments except for ER1 that had a difference of $-0.0004 \%$. It should be noticed that the maximum difference between numerical treatments for $t=900$ (s) is smaller than 0.01 . ER1 tends to 
have a lower front wave depth when compared to the other numerical treatments. ER2 has the lower front wave and ER3 has the highest front wave for the mass conservative numerical treatments. P1 has no mass conservation error and no negative depths as the front wave always adjusts negative depths when they occur.

SWE has the ability to propagate front waves faster that the wave perturbations ahead of it therefore the aforementioned considerations for GWM are not valid for SWE. Oscillations occur throughout the domain. Relative difference is below $10^{-5}$ and is negligible. As in GWM, ER1 has the lower wave front, followed by FR1, ER2, ER3 and the higher is P1. The maximum relative difference is 1 between P1 and FR1 at $t=2700$ (s) when P1 has a cell depth of $2.6 \times 10^{-6}$ and FR1 has no water. Except for this localised point, the relative differences are kept well below 0.01 .

The differences in depth values between the models and analytical solutions were also verified making use of $L^{2}$ :

$$
L^{2}=\sqrt{\frac{\sum_{i=1}^{n}\left(x_{i}^{\text {Num }}-x_{i}^{A n a}\right)^{2}}{\sum_{i=1}^{n} x_{i}^{A n a^{2}}}}
$$

Since the difference between the numerical models and the analytical model are greater than between models, in order to find a difference one has to analyse the 5th significant digit.

Table 3 Comparison of $L^{2}$ depth values between the models and the analytical solution proposed by Hunter et al. (2005)

\begin{tabular}{|c|c|c|c|c|c|c|c|c|}
\hline \multirow[t]{2}{*}{ Model } & \multicolumn{4}{|c|}{ SWE } & \multicolumn{4}{|c|}{ GWM } \\
\hline & $t=900(\mathrm{~s})$ & $t=1800(\mathrm{~s})$ & $t=2700(\mathrm{~s})$ & $t=3600(\mathrm{~s})$ & $t=900(\mathrm{~s})$ & $t=1800(\mathrm{~s})$ & $t=2700(\mathrm{~s})$ & $t=3600(\mathrm{~s})$ \\
\hline ER1 & 0.081517 & 0.061514 & 0.051903 & 0.046052 & 0.025716 & 0.021472 & 0.024725 & 0.028031 \\
\hline ER2 & 0.081519 & 0.061514 & 0.051903 & 0.046054 & 0.025717 & 0.021471 & 0.024724 & 0.028030 \\
\hline ER3 & 0.081521 & 0.061517 & 0.051905 & 0.046057 & 0.025717 & 0.021470 & 0.024722 & 0.028028 \\
\hline $\mathrm{P} 1$ & 0.081521 & 0.061517 & 0.051906 & 0.046056 & 0.025717 & 0.021470 & 0.024722 & 0.028028 \\
\hline FR1 & 0.081520 & 0.061515 & 0.051904 & 0.046054 & 0.025717 & 0.021471 & 0.024724 & 0.028030 \\
\hline
\end{tabular}

\subsection{Non-breaking Wave Run-up on a Planar Beach}

The second test is an adaptation of the first test proposed by Hunter et al. (2005), by introducing a slope in the bed elevation. The test has a domain of $5000(\mathrm{~m}) \times 400(\mathrm{~m})$ and a planar beach slope of $10^{-3}(\mathrm{~m}) /(\mathrm{m})$. It was run with Manning's friction coefficient of $0.01\left(\mathrm{~m}^{1 / 3} \mathrm{~s}^{-1}\right)$. The domain is discretized as 5,273 points and 10,112 cells with an average edge of $21.48(\mathrm{~m})$. The final simulation time was 7200 (s). The inlet boundary condition is a singular positive sine wave with amplitude $8(\mathrm{~m})$, and 7200 (s) period:

$$
h(0, t)=4 \sin (t \pi / 3600)
$$

Results are plotted every 1800 (s) thus showing the beach wetting and drying. Comparison between numerical treatments for GWM and SWE is shown in the top and centre Fig. 2, respectively, and the average surface elevation is plotted in bottom Fig. 2.

Differences of relative magnitude occur in both the GWM and SWE. GWM has more oscillations, mainly upstream. This is the consequence of the combination of two issues: (1) the imposed boundary conditions are those for SWE in both models; (2) GWM has a steeper, slower front wave front when compared to SWE, which, in an ascending run-up might introduce more oscillations. The amount of oscillation is however very small as the maximum difference is roughly $1 \%$. GWM results also show that the wetting of the beach has higher differences than the drying, indicating that the model is more stable during drying processes than during wetting. This is noticeable in the front/rear wave propagation, where the magnitude of the differences between models at $t=1800$ (s) and $t=3600$ (s) is higher than at $t=5400$ (s) and $t=7200$ (s). Moreover, the main instabilities occur near the inlet and during the wetting process. SWE shows a less 


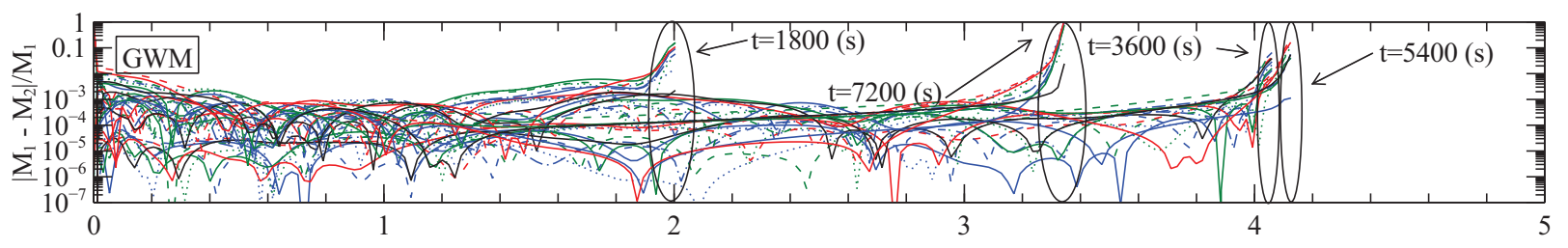

$\mathrm{x}\left(\times 10^{3} \mathrm{~m}\right)$
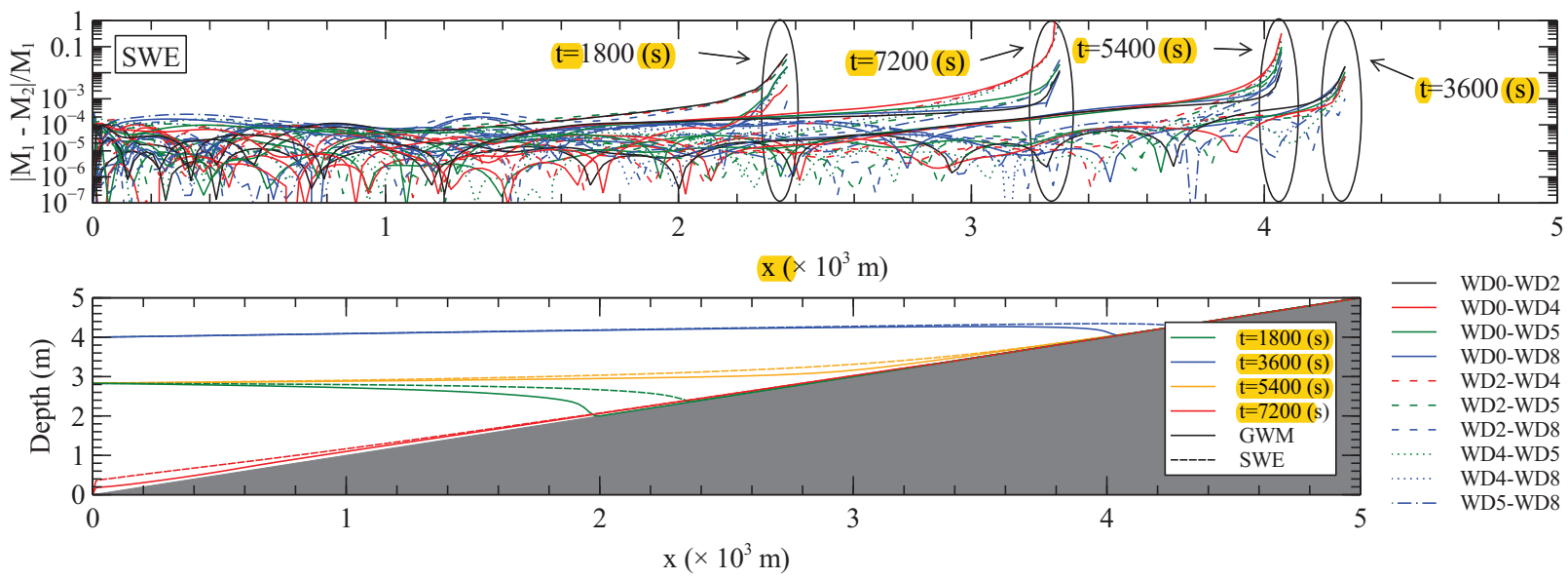

Figure 2 Non-breaking wave run-up on a planar beach centre profile for $t=1800$ (s), $t=3600$ (s), $t=5400$ (s), and $t=7200$ (s). Top: Depth difference between numerical treatments using GWM; Centre: Depth difference between numerical treatments using SWE; Bottom: Free surface position in time for both GWM and SWE.

unstable wetting and drying, with the main differences between numerical treatments at the wave front/rear whether the wave is wetting or drying the beach. Main differences are about $0.1 \%$.

Table 4 summarises the order of numerical treatments from lower to higher peak at the front/rear wave for the time steps selected. ER3, P1, and FR1, although very different numerical treatments, tend to have similar positions relative to each other in both the SWE and GWM. ER2 tends to be the most oscillatory numerical treatment for GWM as it oscillates between the lower and the higher front/rear wave, which is due to the global redistribution.

Table 4 Order of numerical treatments from lower to higher peak at the front/rear wave for the four time steps selected for SWE and GWM

\begin{tabular}{cccccc}
\hline Model & & $t=1800(\mathrm{~s})$ & $t=3600(\mathrm{~s})$ & $t=5400(\mathrm{~s})$ & $t=7200(\mathrm{~s})$ \\
\hline \multirow{6}{*}{ SWE } & Higher & ER2 & ER1 & ER3 & ER3 \\
& $\uparrow$ & P1 & ER3 & P1 & P1 \\
& & FR1 & P1 & FR1 & ER2 \\
& Lower & ER3 & ER2 & ER2 & ER1 \\
\hline \multirow{4}{*}{ GWM } & Higher & P1 & FR1 & ER1 & FR1 \\
& & ER3 & ER2 & ER3 & ER3 \\
& & FR1 & ER3 & PR1 & P1 \\
& Lower & ER2 & P1 & FR1 & ER1 \\
& & ER1 & FR1 & ER2 & FR1 \\
\hline
\end{tabular}

\subsection{Flood propagation over an extended floodplain}

The third test is the propagation of a flood wave over an extended floodplain. This test differs from the nonbreaking wave propagation over an horizontal plane in two aspects. The first difference is in the boundary condition - instead of a stage hydrograph, a flow hydrograph is used. This changes the way SWE and GWM treat the boundary conditions and hence the discrepancies between the two models. The second 
difference is the $2 \mathrm{D}$ radial propagation that is introduced in this test. The test consists of a $(x \times y)=$ $(1000(\mathrm{~m}) \times 2000(\mathrm{~m}))$ domain discretized as 7,563 points and 14,347 cells with an average edge of 10.03 (m). The final simulation time was $10800(\mathrm{~s})$ and the roughness used is $0.05\left(\mathrm{~m}^{1 / 3} \mathrm{~s}^{-1}\right)$. Inlet conditions are imposed at $x=0(\mathrm{~m})$ and $990(\mathrm{~m})<y<1010(\mathrm{~m})$ as the inflow hydrograph presented in Table 5 with intermediary values interpolated. All numerical treatments had absolute global mass conservation within machine precision except ER1 that presented a deficit of 3266 and $4748\left(\mathrm{~m}^{3}\right)$ for GWM and SWE respectively. This represents $1.85 \%$ and $2.68 \%$ relative error, respectively.

Table 5 Flood propagation over an extended floodplain inflow hydrograph

\begin{tabular}{lccc}
\hline $\mathrm{t}(\mathrm{s})$ & 300 & 3600 & 10800 \\
\hline Inflow $\left(\mathrm{m}^{3} / \mathrm{s}\right)$ & 0 & 20 & 20 \\
\hline
\end{tabular}

Results obtained for the central profile are presented in Fig. 3 and the 2D overview of the differences in Fig. 4.

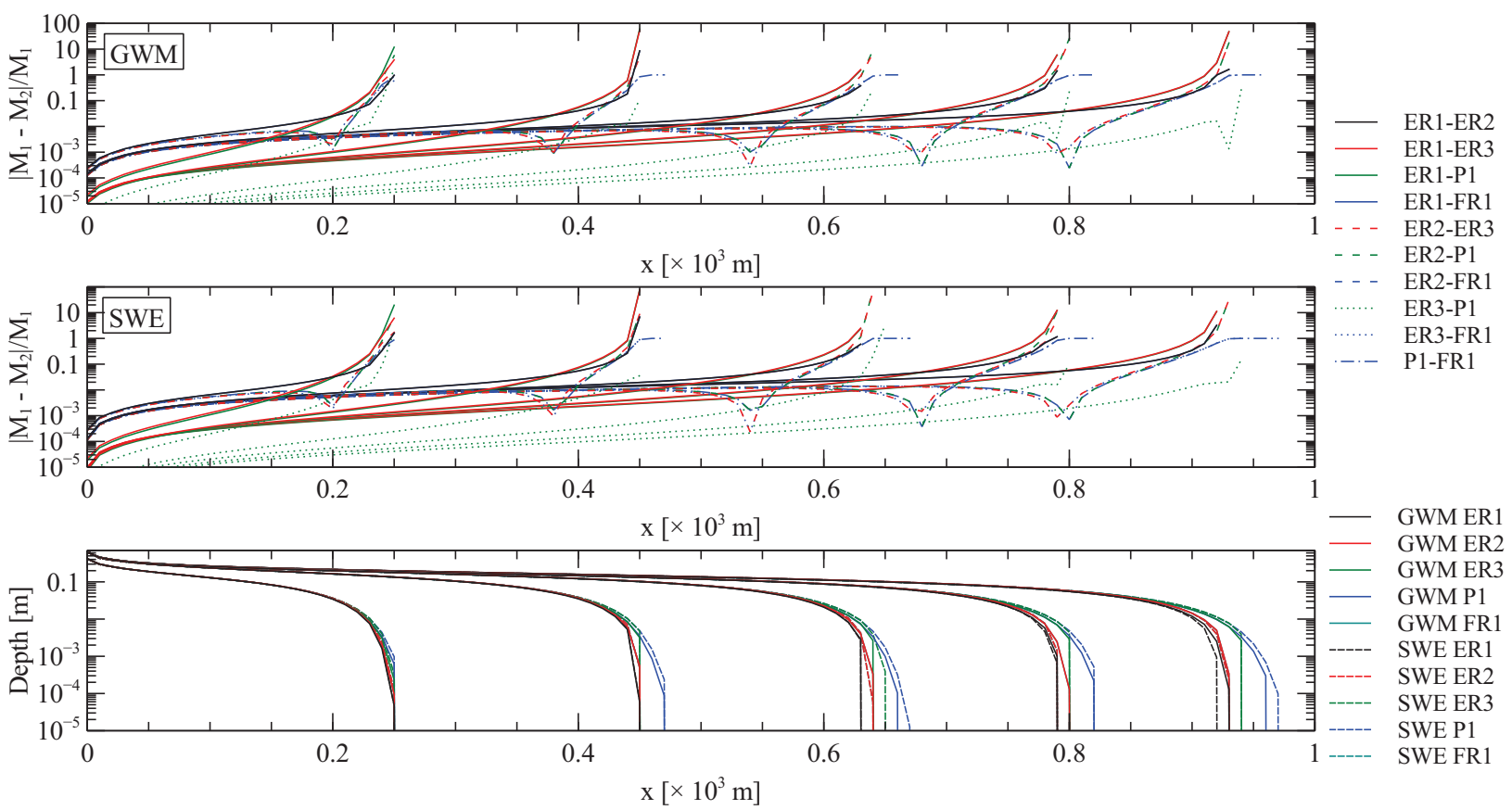

Figure 3 Flood propagation over an extended floodplain centre $(y=1000(\mathrm{~m}))$ profile for $t=1800(\mathrm{~s}), t=3600(\mathrm{~s}), t=5400(\mathrm{~s}), t=7200(\mathrm{~s})$, and $t=9000$ (s). Left: Difference between numerical treatments using GWM; Centre: Difference between numerical treatments using SWE; Right: Free surface for all WD numerical treatments for both GWM and SWE.

Figure 3 shows in the top the comparison between numerical treatments for the GWM, on the centre the comparison for SWE and on the bottom the surface profile for the two models. In Figure 3, unlike the non-breaking wave propagation over an horizontal plane and the non-breaking wave run-up on a planar beach, there are no oscillations in the boundary condition as the values are not imposed as in previous tests. Differences between the WD numerical treatments are very similar between the two models. ER3 is the numerical treatment with the higher peak difference in surface level with a very similar value to P1, and ER1 is the lowest. P1 is the faster front wave followed by ER3 whilst the ER1 is slower for both SWE and GWM. Differences are up to $0.01(\mathrm{~m})$ which, translated to relative difference can go as high as 100 . As seen by the top and centre image the main difference between GWM and SWE WD numerical treatments is the magnitude of the difference as the lines are very similar. FR1 and ER2 have the same results, as explained in the non-breaking wave propagation over an horizontal plane, due to non-existence of negative depths. 
Again, ER3 and P1, although different in concept have a very high degree of similitude.

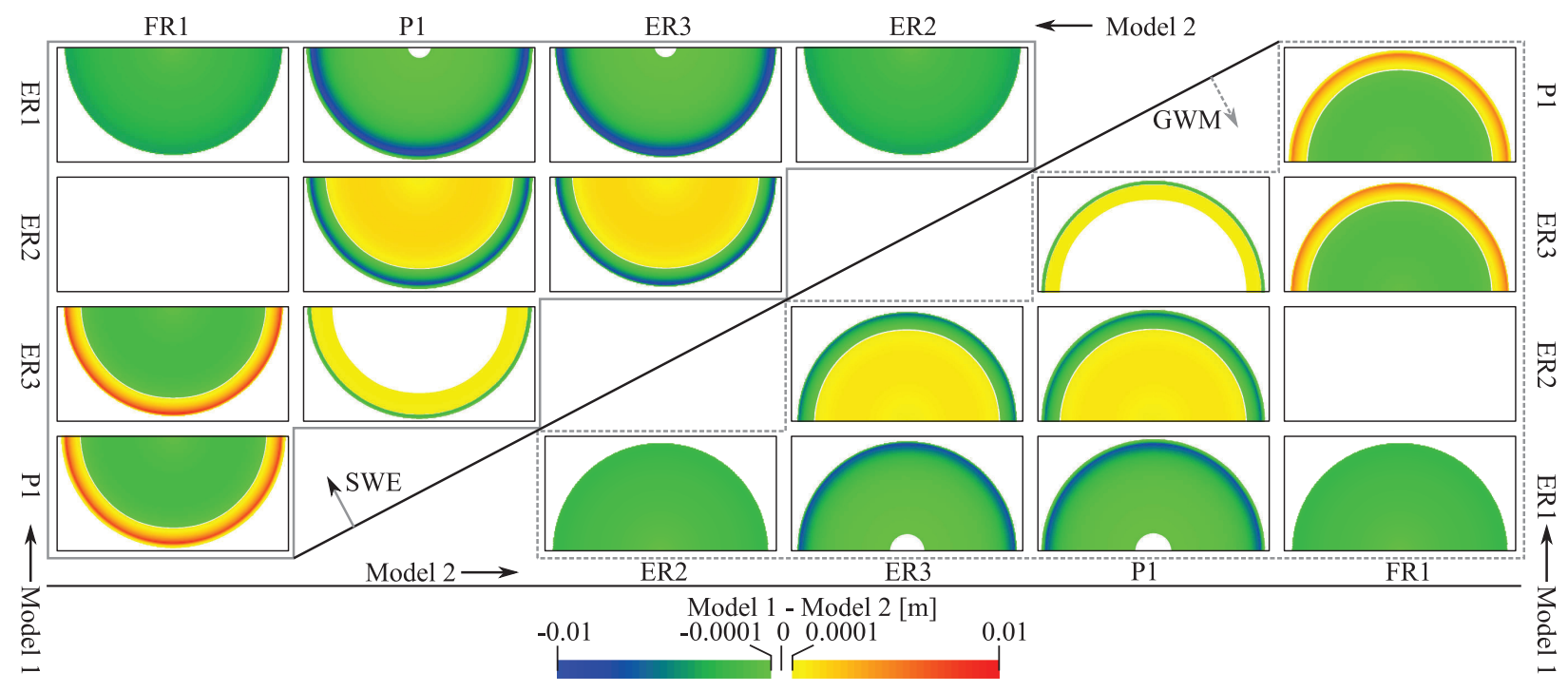

Figure 4 Depth differences between WD numerical treatments for the flood propagation over an extended floodplain for GWM and SWE. GWM representation in the lower triangle, SWE representation in the upper triangle; Model 1 vertical and Model 2 horizontal.

Figure 4 shows the difference in simulated depth between all the numerical treatments for the two models studied: SWE and GWM. ER1 mass conservation error is visible through the difference between ER1 and the other treatments, as the depth is always below the remaining numerical treatments. ER2 shows a lower front wave and a higher depth behind the front wave as expected since the water is redistributed along the whole domain. ER3 shows a lower depth in the front wave and a slightly higher depth behind it. The rest of the domain is similar between ER3 and P1. All the numerical treatments keep a very high degree of radial symmetry. FR1 cannot be evaluated by this test as no negative depth occur and therefore is not activated.

\subsection{Valley flooding}

The last test is the Valley flooding (Néelz \& Pender, 2012). The test consists of a river valley with length of approximately $17000(\mathrm{~m})$ and a width of $800(\mathrm{~m})$ with slopes between $1 \%$ upstream and $0.1 \%$ downstream. All the boundaries are reflective except for the upstream inlet with length of $260(\mathrm{~m})$. The inflow hydrograph is a skewed trapezoid with $3000\left(\mathrm{~m}^{3} / \mathrm{s}\right)$ peak (Table 6). Both super and subcritical flows occur in the simulation. The simulation runs for 54000 (s) with an average cell edge size of 73.9 (m) (7,562 nodes), and a constant $0.04\left(\mathrm{~m}^{1 / 3} \mathrm{~s}^{-1}\right)$ roughness value.

Table 6 Valley flooding inflow hydrograph

\begin{tabular}{lcccc}
\hline$t(\mathrm{~s})$ & 300 & 600 & 1200 & 6000 \\
\hline Inflow $\left(\mathrm{m}^{3} / \mathrm{s}\right)$ & 0 & 3000 & 3000 & 0 \\
\hline
\end{tabular}

$C_{m}$ coefficient is used to compare all modelling results:

$$
C_{m}=\frac{c_{w, w}-\left(c_{w, d}+c_{d, w}\right)}{c_{w, w}+c_{w, d}+c_{d, w}}
$$

where, $c_{w, w}$ is the number of wet cells common to all models, $c_{w, d}$ the number of wet cells in the model that are dry in any of the other models, and $c_{d, w}$ the number of dry cells that are wet in any of the other models. The $C_{m}$ coefficient ranges from -1 to 1 , where 1 is a perfect fit, 0 when the number of correctly and erroneously predicted cells is equal, and -1 for the case when all cells differ. Results for all models and 
all numerical treatment are presented in Table 7. It should be noticed that the coefficient only provides a degree of agreement between all of the treatments and not an absolute comparison.

$\begin{aligned} & \text { Table } 7 \\
& \text { ments }\end{aligned}$
\begin{tabular}{lccccc} 
Model $C$ & ER1 & ER2 & ER3 & P1 & FR1 \\
\hline SWE & 0.7266 & 0.8549 & 0.9906 & 0.9795 & 0.945 \\
GWM & 0.7056 & 0.8262 & 0.9926 & 0.9760 & 0.9562 \\
\hline
\end{tabular}

Table 7 shows an excellent agreement for ER3, P1 and FR1, whilst ER1 and ER2 do not perform so well. This comparison shows that ER3 is the model that predicts less erroneous wet or dry cells.

Figure 5 shows the contour for $h=0.001(\mathrm{~m})$ for all the numerical treatments used (i.e. ER1, ER2, ER3, $\mathrm{P} 1, \mathrm{FR} 1$ ) and models (i.e. SWE and GWM) for both the front and rear waves at $t=9000$ (s). SWE results are presented at the top and GWM at the bottom. All models present a somewhat similar result. Overlapping of contours happens upstream, where small ponds are left behind due to the topography. ER1 clearly shows a larger contour, upstream and downstream, due to the excess of water erroneously introduced in the simulation.

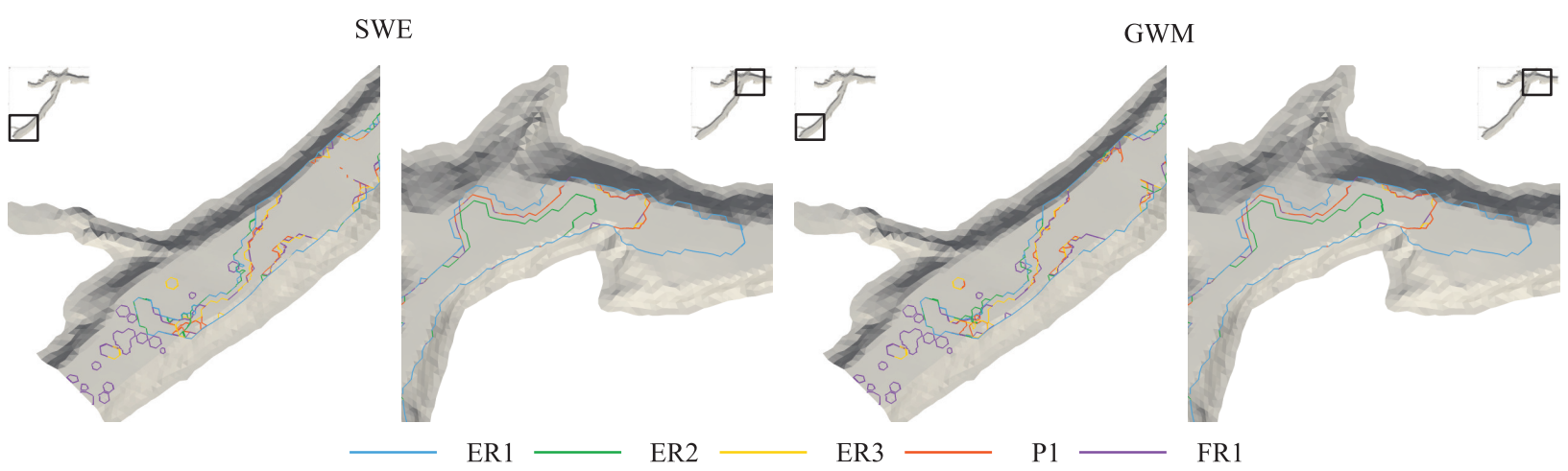

Figure 5 Contour for $h=0.001(\mathrm{~m})$ for all the numerical treatments used (i.e. ER1,ER2,ER3,P1,FR1) and models (i.e. SWE and GWM) for both the front and rear waves at $t=9000(\mathrm{~s})$. SWE results are presented on the top and GWM on the bottom.

ER1 stands out with the largest differences with all other numerical treatments, because it adds water in the rear waves that become negative both for GWM and SWE. ER1 has a mass error of $96 \%$ and $98 \%$ for the GWM and SWE, respectively. ER1 is deemed completely incorrect and therefore inadequate for this simulation since it doubled the volume of water in the domain. ER1 and ER2 differ in some points for more than 1 metre from the rest of numerical treatments. ER2 tends to have a slower front wave, with a greater depth upstream, as seen in Fig. 5, a clear result of the global redistribution of the volume to upstream cells. $\mathrm{P} 1$ has a mass error of $1.08 \%$ and $1.19 \%$ for the GWM and SWE respectively. This value is much smaller than ER1. ER3 has a very similar result to P1 and FR1 with a slightly lower wave front than P1. FR1 has a different behaviour between GWM and SWE since in GWM the front wave is higher whilst in SWE it is lower when compared to ER3 and P1. The difference between numerical treatments clearly shows that the choice of WD numerical treatment is of the utmost importance as a simple treatment of the WD front can lead to very different results given complex flows and geometries.

\section{Conclusions}

A novel wetting-drying (WD) numerical treatment (ER3) was proposed and thoroughly tested by applying it to an approximate Roe Riemann solver. Testing was done for four flow situations by comparing differences between pairs of WD numerical treatments including the novel numerical treatment and four numerical treatments known from literature adapted to SWE and GWM equations. WD numerical treatments were 
shown to be of the utmost importance as a small difference in the WD numerical treatment can lead to large errors in the waves' propagation. A simple adjustment of the negative depths obtained from the numerical calculations is not sufficient as it can lead to large mass errors $(\approx 200 \%$ in the simulation made). Simple element removal global distributing WD numerical treatments can introduce errors and degenerate the solution transporting volume of water upstream. Overall local correction WD numerical treatments (ER3) and flux restricting numerical treatments (FR1) have shown the best results. Although with mass conservation errors the negative depth numerical treatment (P1) provided similar results to the local correction numerical treatment and flux restricting numerical treatment.

The presented set of simulations indicate that the novel WD numerical treatment based on the "gravitational" attraction coefficient presented in this paper (ER3) shows at least similar performance to FR1 without the need to compute twice the fluxes, which usually translates into a lower computational time and is overall superior to all other tested numerical treatments.

\section{Funding}

This research is partially funded by the FCT (Portuguese Foundation for Science and Technology) through the Doctoral Grant SFRH/BD/81869/2011 financed through the POPH/FSE (Programa Operacional Potencial Humano/Fundo Social Europeu) program. This study had the support of Portuguese Foundation for Science and Technology (FCT) Project UID/MAR/04292/2013. 


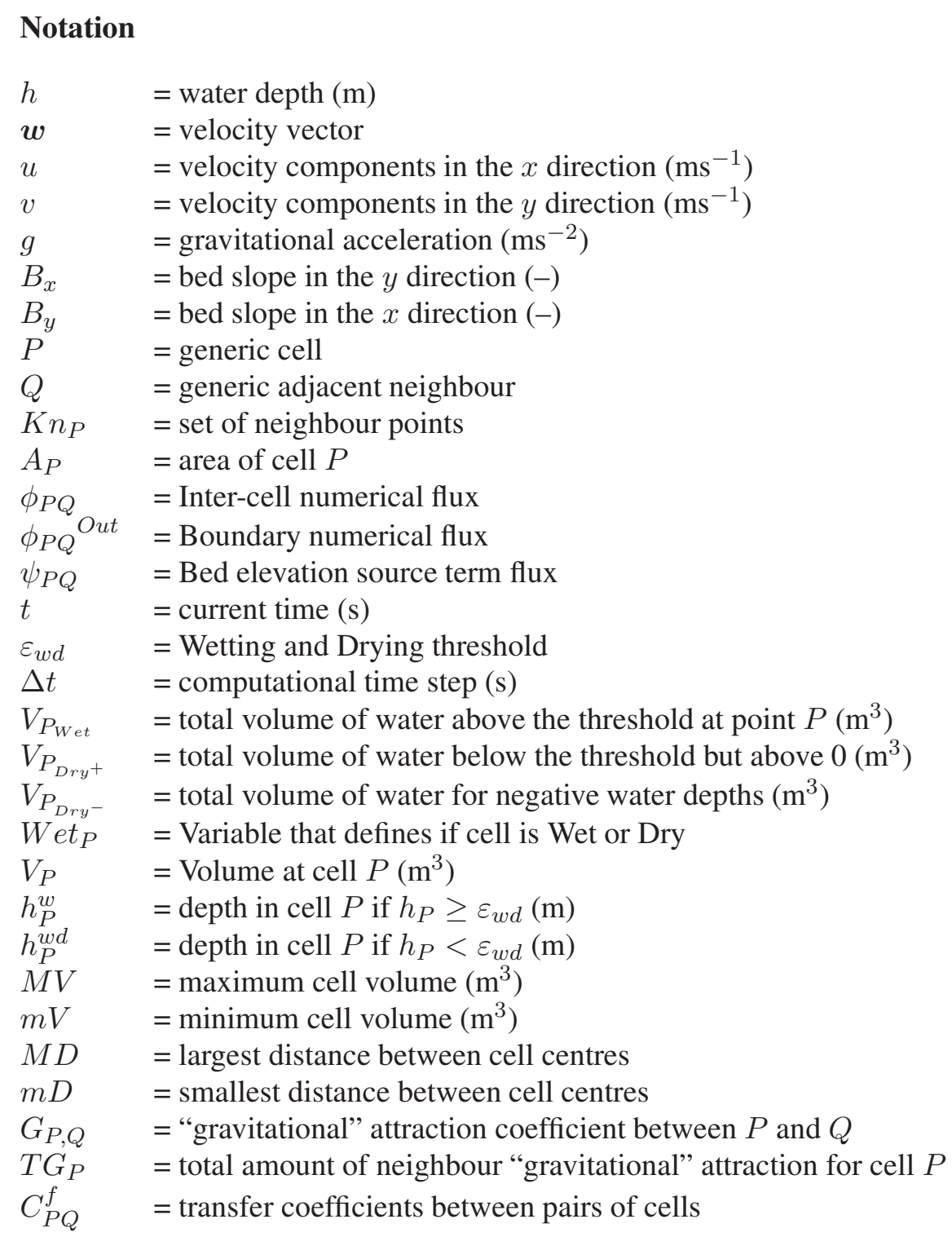

\section{References}

Audusse, E., \& Bristeau, M.-O. (2005). A well-balanced positivity preserving second-order scheme for shallow water flows on unstructured meshes. Journal of Computational Physics, 206(1), 311-333. doi:

Aureli, F., Maranzoni, A., Mignosa, P., \& Ziveri, C. (2008). A weighted surface-depth gradient method for the numerical integration of the $2 \mathrm{D}$ shallow water equations with topography. Advances in Water Resources, 31, 962-974. doi:

Bates, P., \& Hervouet, J.-M. (1999). A new method for moving-boundary hydrodynamic problems in shallow water. Proceedings of the Royal Society A: Mathematical, Physical and Engineering Sciences, 455(1988), 3107-3128. doi:

Begnudelli, L., \& Sanders, B. (2006). Unstructured grid finite-volume algorithm for shallow-water flow and scalar transport with wetting and drying. Journal of Hydraulic Engineering(April), 371-384.

Brufau, P., García-Navarro, P., \& Vázquez-Cendón, M. (2004). Zero mass error using unsteady wettingdrying conditions in shallow flows over dry irregular topography. International Journal for Numerical Methods in Fluids., 45(May), 1047-1082. doi: 
Brufau, P., Vázquez-Cendón, M., \& García-Navarro, P. (2002). A numerical model for the flooding and drying of irregular domains. International Journal for Numerical Methods in Fluids., 39(October), 247-275. doi:

Buffard, T., Gallouët, T., \& Hérard, J.-M. (2000). A sequel to a rough Godunov scheme: application to real gases. Computers \& Fluids, 29, 813-847. doi:

Castro, M., Ferreiro Ferreiro, A., García-Rodríguez, J., González-Vida, J., Macías, J., Parés, C., \& VázquezCendón, M. (2005). The numerical treatment of wet/dry fronts in shallow flows: application to one-layer and two-layer systems. Mathematical and Computer Modelling, 42, 419-439. doi:

Duran, A., Liang, Q., \& Marche, F. (2013). On the well-balanced numerical discretization of shallow water equations on unstructured meshes. Journal of Computational Physics, 235, 565-586. doi:

Einfeldt, B., Munz, C., Roe, P., \& Sjögreen, B. (1991). On Godunov-type methods near low densities. Journal of Computational Physics, 92, 273-295.

Godunov, S., Zabrodin, A., \& Prokopov, G. (1961). A computational scheme for two-dimensional nonstationary problems of gas dynamics and calculation of the flow from a shock wave approaching a stationary state. Zhurnal Vychislitel'noi Matematiki i Matematicheskoi Fiziki, 1(6), 1020-1050.

Harten, A. (1983). High resolution schemes for hyperbolic conservation laws. Journal of Computational Physics, 49(3), 357-393. doi:

Heniche, M., Secretan, Y., Boudreau, P., \& Leclerc, M. (2000). A two-dimensional finite element dryingwetting shallow water model for rivers and estuaries. Advances in Water Resources, 23(4), 359-372. doi:

Hof, B., \& Vollebregt, E. (2005). Modelling of wetting and drying of shallow water using artiÿcial porosity. International Journal for Numerical Methods in Fluids, 48, 1199-1217. doi:

Hunter, N., Horritt, M., Bates, P., Wilson, M., \& Werner, M. (2005). An adaptive time step solution for raster-based storage cell modelling of floodplain inundation. Advances in Water Resources, 28(9), 975-991. doi:

Kärnä, T., de Brye, B., Gourgue, O., Lambrechts, J., Comblen, R., Legat, V., \& Deleersnijder, E. (2011). A fully implicit wettingdrying method for DG-FEM shallow water models, with an application to the Scheldt Estuary. Computer Methods in Applied Mechanics and Engineering, 200(5-8), 509-524. doi:

Leandro, J., Chen, A., \& Schumann, A. (2014). A 2D parallel diffusive wave model for floodplain inundation with variable time step (P-DWave). Journal of Hydrology, 517, 250-259. doi:

Leandro, J., Schumann, A., \& Pfister, A. (2016). A step towards considering the spatial heterogeneity of urban key features in urban hydrology flood modelling. Journal of Hydrology, 535, 356 - 365. doi:

Liang, Q., \& Marche, F. (2009). Numerical resolution of well-balanced shallow water equations with complex source terms. Advances in Water Resources, 32(6), 873-884. doi:

Martins, R., Leandro, J., \& Djordjević, S. (2015). A well balanced Roe Scheme for the local inertial equations with an unstructured mesh. Advances in Water Resources, 83, 351-363. doi:

Martins, R., Leandro, J., \& Djordjević, S. (2016). Influence of sewer network models on urban flood damage assessment based on coupled 1d/2d models. Journal of Flood Risk Management, n/a-n/a. doi:

Medeiros, S., \& Hagen, S. (2013). Review of wetting and drying algorithms for numerical tidal flow models. International Journal for Numerical Methods in Fluids(71), 473-487. doi:

Murillo, J., \& García-Navarro, P. (2010). Weak solutions for partial differential equations with source terms: Application to the shallow water equations. Journal of Computational Physics, 229(11), 4327-4368. doi:

Murillo, J., García-Navarro, P., Burguete, J., \& Brufau, P. (2006). A conservative 2D model of inundation flow with solute transport over dry bed. International Journal for Numerical Methods in Fluids, 52(10), 1059-1092. doi:

Neal, J., Villanueva, I., Wright, N., Willis, T., Fewtrell, T., \& Bates, P. (2012). How much physical complexity is needed to model flood inundation? Hydrological Processes, 26(15), 2264-2282. doi:

Néelz, S., \& Pender, G. (2012). Benchmarking of 2D hydraulic modelling packages (Tech. Rep.). Bristol: 
1

2

Environment Agency.

Nikolos, I., \& Delis, A. (2009). An unstructured node-centered finite volume scheme for shallow water flows with wet/dry fronts over complex topography. Computer Methods in Applied Mechanics and Engineering, 198(47-48), 3723-3750. doi:

Song, L., Zhou, J., Guo, J., Zou, Q., \& Liu, Y. (2011). A robust well-balanced finite volume model for shallow water flows with wetting and drying over irregular terrain. Advances in Water Resources, 34(7), 915-932. doi:

Xing, Y., \& Shu, C. W. (2011). High-order finite volume WENO schemes for the shallow water equations with dry states. Advances in Water Resources, 34(8), 1026-1038. doi: 

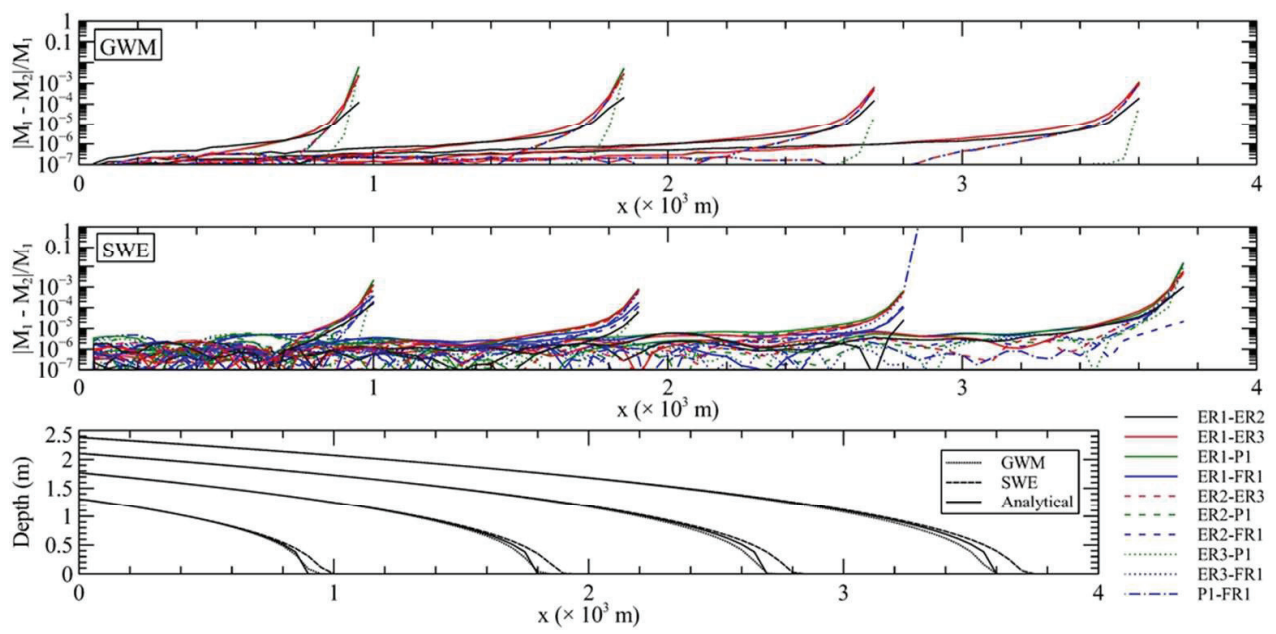

Non-breaking wave propagation over an horizontal plane for $\$ \mathrm{t}=\$ 900(\mathrm{~s}), \$ \mathrm{t}=\$ 1800(\mathrm{~s}), \$ \mathrm{t}=\$ 2700$ (s) and $\$ t=\$ 3600$ (s). Top: relative depth difference between numerical treatments using GWM; Centre: relative depth difference using SWE; Bottom: Free surface position for the analytical solution, GWM, and SWE. Fig. 1

$90 \times 45 \mathrm{~mm}(300 \times 300 \mathrm{DPI})$ 

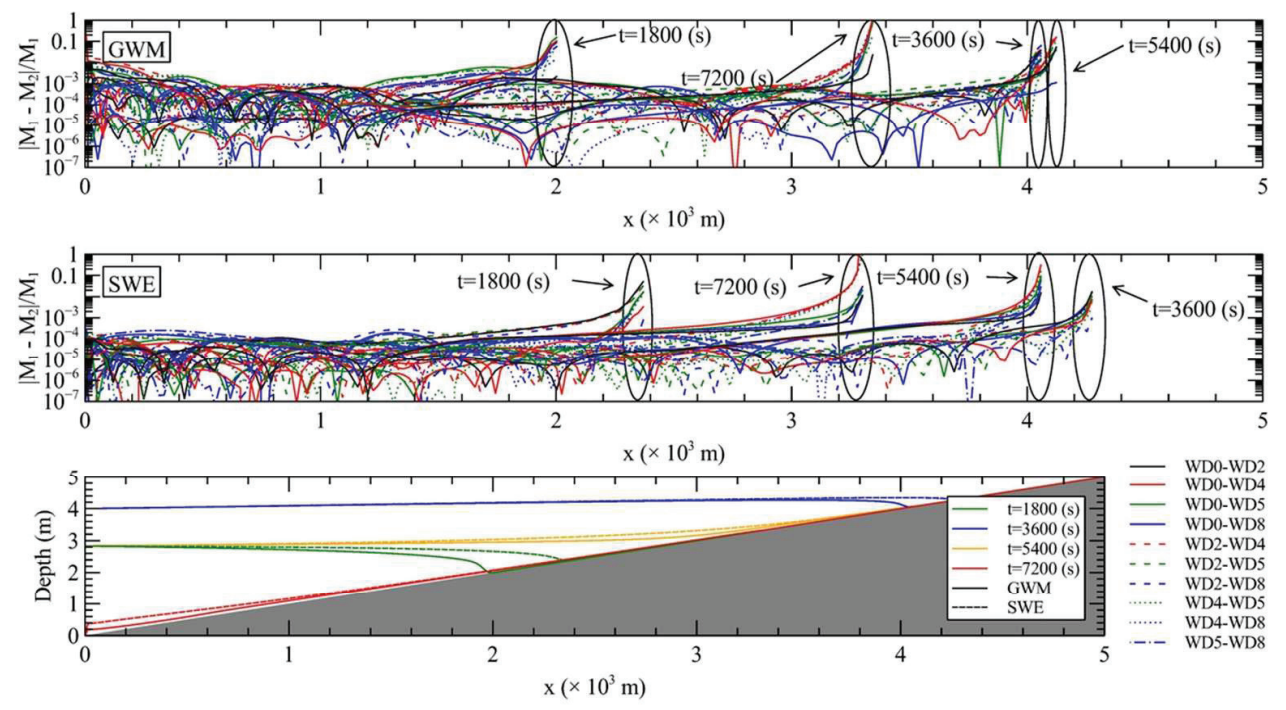

Non-breaking wave run-up on a planar beach centre profile for $t=1800(\mathrm{~s}), \mathrm{t}=3600(\mathrm{~s}), \mathrm{t}=5400(\mathrm{~s})$, and $t=7200$ (s). Top: Depth difference between numerical treatments using GWM; Centre: Depth difference between numerical treatments using SWE; Bottom: Free surface position in time for both GWM and SWE. Fig. 2

$100 \times 55 \mathrm{~mm}(300 \times 300$ DPI $)$ 


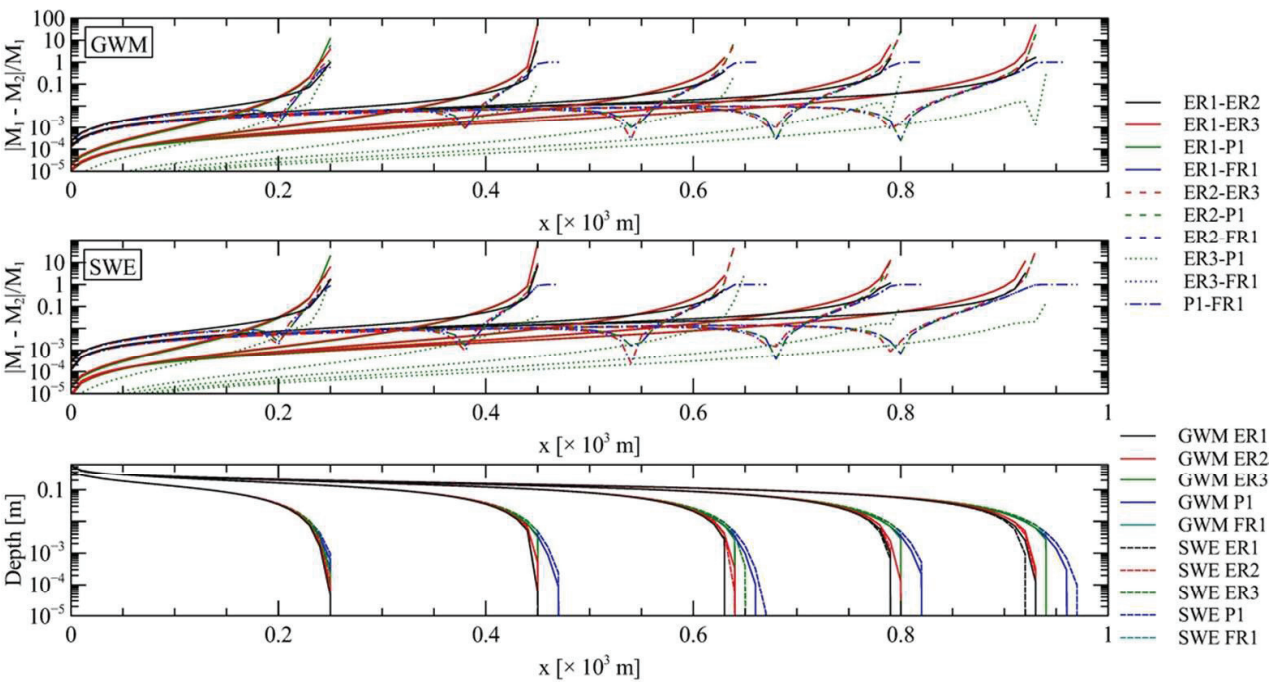

Flood propagation over an extended floodplain centre $(y=1000(\mathrm{~m}))$ profile for $t=1800(\mathrm{~s}), \mathrm{t}=3600(\mathrm{~s})$, $\mathrm{t}=5400(\mathrm{~s}), \mathrm{t}=7200$ (s), and $\mathrm{t}=9000$ (s). Left: Difference between numerical treatments using GWM; Centre: Difference between numerical treatments using SWE; Right: Free surface for all WD numerical treatments for both GWM and SWE.

$100 \times 55 \mathrm{~mm}(300 \times 300$ DPI $)$ 


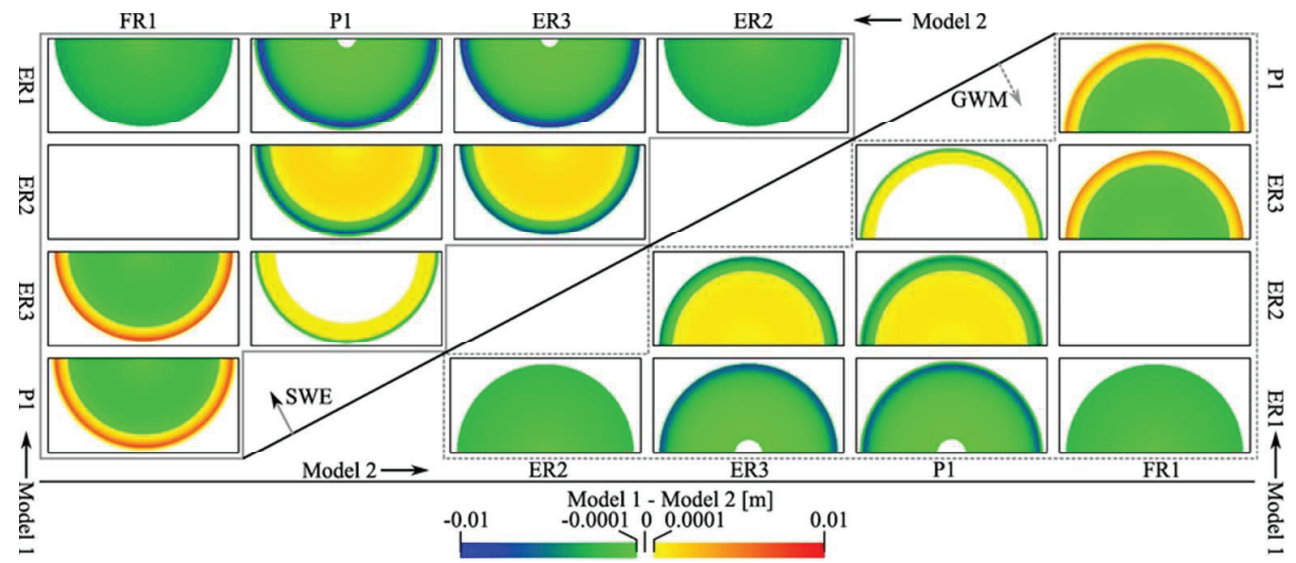

Depth differences between WD numerical treatments for the flood propagation over an extended floodplain for GWM and SWE. GWM representation in the lower triangle, SWE representation in the upper triangle; Model 1 vertical and Model 2 horizontal.

$$
\text { Fig. } 4
$$

$77 \times 33 \mathrm{~mm}(300 \times 300 \mathrm{DPI})$ 


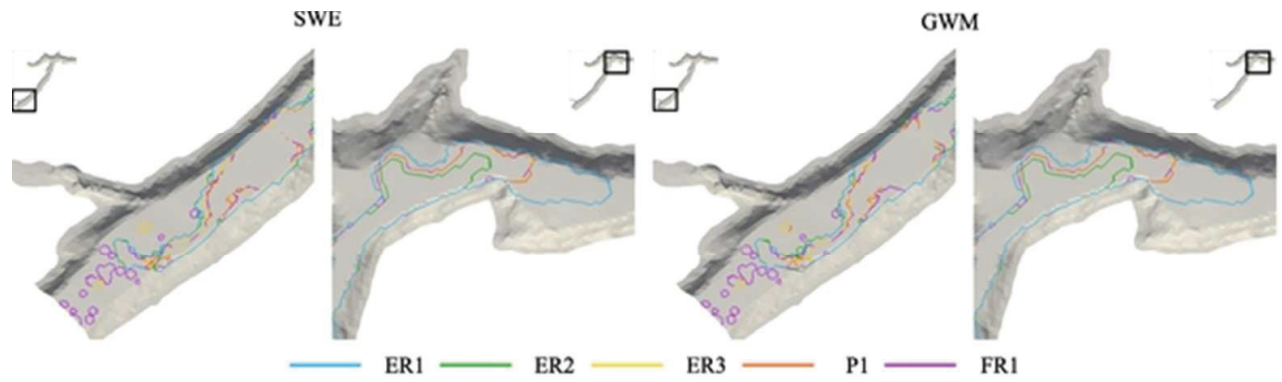

Contour for $h=0.001(\mathrm{~m})$ for all the numerical treatments used (i.e. ER1,ER2,ER3,P1,FR1) and models (i.e. SWE and GWM) for both the front and rear waves at $t=9000(\mathrm{~s})$. SWE results are presented on the top and GWM on the bottom.

$$
\text { Fig. } 5
$$

$51 \times 14 \mathrm{~mm}(300 \times 300 \mathrm{DPI})$ 